\title{
A Spatial Information Platform for Urban and Rural Planning based on One Map
}

\author{
a.F. ZHU, Q.W. ZHANG, X.F. LIU \& F. YAN, ${ }^{b}$ Z. TONG \\ ${ }^{a}$ School of Hydropower and Information Engineering, Huazhong University of Science and \\ Technology, China \\ ${ }^{\mathrm{b}}$ Technical Development Center, $\operatorname{CCCC}($ China Communications Construction Company) Second \\ Harbor Consultants Co.,Ltd., China
}

\begin{abstract}
KEYWORD: Urban and rural planning; Spatial information platform; Software system; One Map; GIS.

ABSTRACT: The organization and utilization of information resources are emphasis and difficulty for urban and rural planning management. In addition to traditional text data, there are massive and multi-source heterogeneous spatial data. It is necessary to build an integrated platform to manage these data. In order to improve the efficiency of management of urban and rural planning, the concept of One Map is introduced in this paper. By combining One Map with Geographic Information Systems (GIS), an Urban and Rural Planning Spatial Information Platform (URPSIP) is designed and developed. URPSIP is based on Client/Server structure. Spatial data is stored in Oracle relational database, and accessed through ArcSDE, which is a spatial database engine. URPSIP could provide a visual working platform to browse, query, analyze and share data expediently in one map. Finally, a case study in Wuhan indicated that URPSIP is able to significantly integrate current resources of planning and improve the efficiency of management of urban and rural planning.
\end{abstract}

\section{INTRODUCTION}

Urban planning is prerequisite for urban development. It is significant to ensure a scientific and sustainable growth of a city. With sustained and rapid urbanization in China, urban population has increased and urban scale has expanded. Traditional dual structure with city and country has become an integrated urban-rural structure [1]. So, integration management is the main challenges facing urban and rural planning in the current situation.

The core of urban and rural planning is data, particularly spatial data. Therefore, the key problem is how to organize and utilize these massive and multi-source heterogeneous spatial data efficiently [2].

Geographic Information System (GIS) is a kind of computer system. It can provide a solution to capture, store, display, edit, analyze, and share all types of spatial or geographical data. For this reason, GIS has been widely applied in many fields, particularly in urban and rural planning [3, 4].

GIS can improve the data management of urban and rural planning. However, there are still some problems as follows: (1) Planning results are numerous and disorderly. It is hard to avoid contradictions between different versions. (2) Planning resources are usually scattered among different organizations or departments. It is difficult to manage and maintain. (3) Efficiency reduced because of information asymmetry.

In order to solve these problems, the concept of One Map is introduced in this paper. By combining One Map with GIS, an integrated platform of Urban and Rural Planning Spatial Platform (URPSIP) is designed and developed.

\section{CONCEPT OF ONE MAP}

\section{Definition of One Map}

One Map aims integrate all planning information in one system, like in one map. It can be regarded as following three respects [5]. 
Firstly, One Map is based on the current data. The current geographic data is the foundation for urban and rural planning. In terms of content of data, the current geographic data includes land use, cadaster, terrain and image. In terms of format of data, it includes vector, raster and text. One Map is able to store and organize these multi-source heterogeneous data uniformly.

Secondly, One Map is limited by the regulatory plan. The regulatory plan provides rules to limit land status, land use intensity and space environment control. It is approved with legal effect, and an important reference for planning management. So, the regulatory plan is the core of One Map.

Finally, One Map is an integrated working platform. It provides a highly integrated working system to manage, analyze, update and share all types of planning data.

\section{Logical framework of One Map}

The logical framework of One Map includes four sub-maps (Fig. 1).

(1) The Current Map includes land use maps, cadaster maps, terrain maps and images. It also includes urban three-dimensional data. The Current map describes basic information of city.

(2) The Core Map includes master plans, regulatory plans, statutory plans, spatial control maps, etc. It provides the rules for planning.

(3) The Managerial Map includes planning formulations, planning approvals, planning supervisions, etc. The Managerial map is a business-oriented dataset, which is the most important part in planning management.

(4) The Product Map refers to achievements of planning such as immediate plans.It also includes results of special planning such as transportation plans and tourism plans.

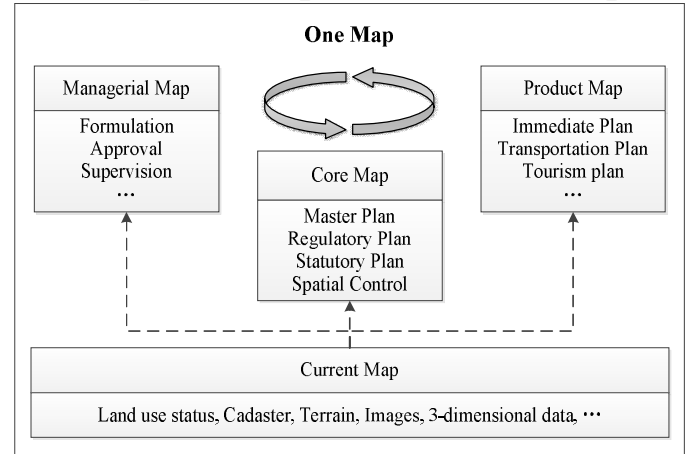

Figure 1. The Logical framework of One Map

\section{REQUIREMENT ANALYSIS AND SYSTEM DESIGN}

\section{Requirement of URPSIP}

URPSIP aims to design and develop an integrated system to manage multi-source heterogeneous data as a whole. Specifically, URPSR was required as follows.

(1) URPSIP needs to store and organize all types of data uniformly.

(2) URPSIP needs to display, manipulate and analyze planning data through graphics and its attribute synchronously.

(3) URPSIP needs to support the business of planning, such as drawing and printing maps.

(4) URPSIP needs to exchange and share planning information between different networks and systems.

\section{Design of system architecture}

URPSIP adopt a kind of three-layer C/S architecture as in Fig. 2. On the one hand, C/S architecture can be constructed under the local area network (LAN), which is faster and more security. On the other hand, this three-layer architecture can balance system loads between server and client to improve the overall performance of the system. 


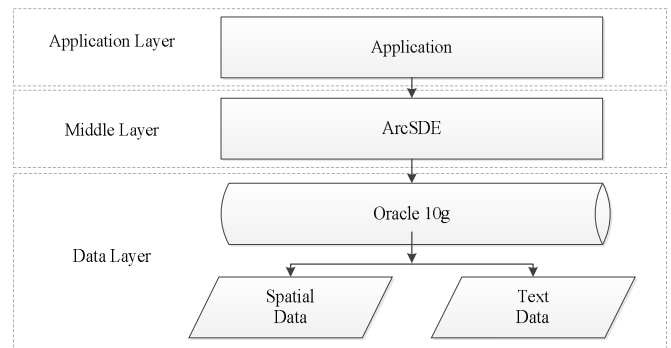

Figure 2. System architecture of URPSIP

In the data layer in Fig. 2, massive amounts of planning data are stored by relational databases such as Oracle 10g. So, the spatial data such as graphics and image cannot be accessed directly. Thus a spatial database engine (SDE) server is deployed in the middle layer. The ArcSDE server is a translator to be a bridge between the data layer and the application layer. In addition, ArcSDE server is able to support massive data [6]. The application layer is in the top of the system architecture. Applications in the application layer can access multi-source heterogeneous spatial data through ArcSDE server in undifferentiated ways.

\section{Design of function modules}

In order to satisfy the requirements of the system, URPSIP would be split into 7 essential function modules as in Fig. 3. These modules are not only independent from each other, but also associated with each other.

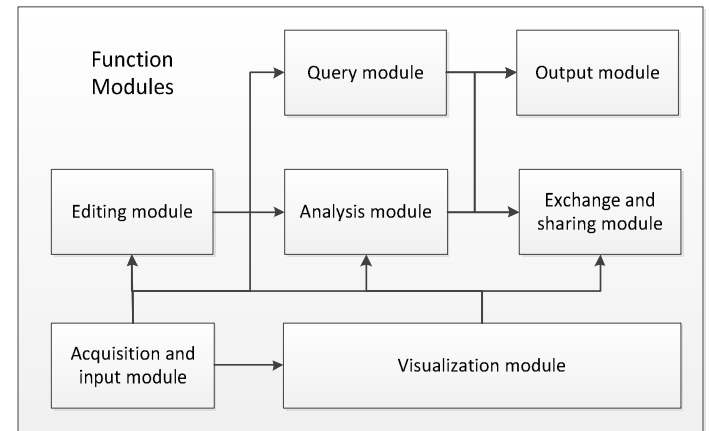

Figure 3. Function Modules of URPSIP

\section{Data acquisition and input module}

This module is designed to obtain multi-source heterogeneous data and import them into URPSIP. The text data would be stored as relationship table, and the spatial data, such as graphics and images, would be accessed by ArcSDE. The spatial data must be with the same coordinates before they are put in the storage.

\section{Editing module}

This module is designed to manipulate and modify data in URPSIP, such as insert, update and delete a text record. In addition, this module can add, move, alter and delete geographic features like CAD.

\section{Visualization module}

This module is designed to support displaying all sorts of maps in one map. It can zoom in and out to browse the map, and render each geographic layer or feature by custom symbols.

\section{Query module}

This module is designed to query data from relational or spatial database. Querying data from text table can be executed by SQL. To geographic features, this module support locating geographic features by their attribute and topology relations.

\section{Analysis module}

This module designs many spatial analysis methods to analyze spatial data, such as buffer analysis and overlay analysis. Meanwhile, this module can calculate some essential statistic values, such as mean, median and variance. 


\section{Output module}

This module is designed to export planning results to the disk as the picture format such as .jpg, or print these maps directly after appending other elements (e.g. compass, scale and legend) to the maps.

\section{Exchange and sharing module}

This module is designed to send planning data to other databases or Web servers, and access data from other servers.

\section{DEVELOPMENT AND APPLICATION}

\section{Development environment}

URPSIP was developed based on component GIS, which is a kind of redevelopment method for GIS. The component GIS packages underlying data structures and methods as various components. So developers utilize these components to build GIS systems. This redevelopment method based on component GIS is flexible, robust, cost-efficient and extensible, and helps developers focus on how to accomplish functions without worrying underlying data structures. It is a popular method in software development of GIS.

In this paper, the GIS component was chosen as ArcEngine. Visual Studio 2008 is adopted as IDE. The programing language is Visual Basic.net. At the server side, text spatial data are stored in Oracle 10g. An ArcSDE server is also deployCed upon the Oracle database to organize and access spatial data.

\section{Study area}

Wuhan $\left(113^{\circ} 41^{\prime} \sim 115^{\circ} 05^{\prime} \mathrm{E}, 2^{\circ} 58^{\prime} \sim 31^{\circ} 22^{\prime} \mathrm{N}\right)$ is the capital of Hubei province, and is located in the eastern Jianghan Plain in the central China. Wuhan consists of 13 districts. The total area is $8494.14 \mathrm{~km} 2$. As of 2014, the total population of Wuhan is over 10,338,000. Wuhan is one of the most important cities in the central China.

\section{A case study in Wuhan}

Fig. 4 shows the main interface of URPSIP in Wuhan. After connecting to the Oracle database, all of planning data, such as basic geographic data, regulatory plans or other planning results, could be added to the map view on the main interface. Meanwhile, users can process these maps and print them in the layout view. Through the layer management view on the left of the main interface, users can decide whether a layer display in the map view. Using menus and tools on the top, users could conveniently browse maps, edit features, search location and attribution of spatial data, analyze layers spatially and share information with other systems.

So, URPSIP truly achieved the integration management of urban and rural planning in one map.

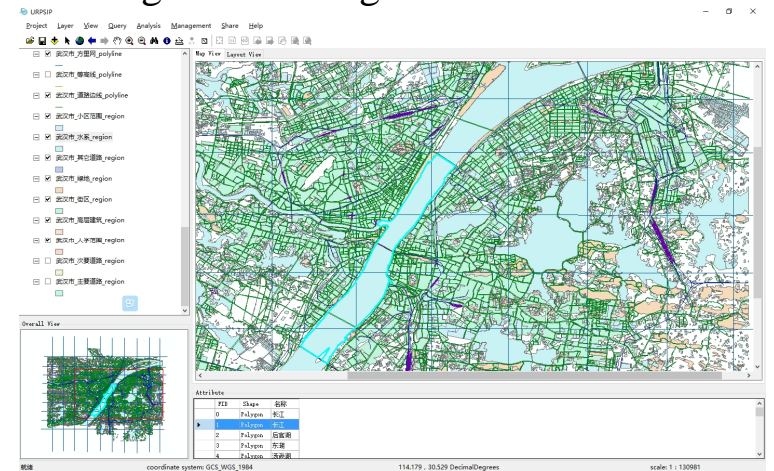

Figure 4.The main interface of URPSIP

\section{CONCLUSION}

URPSIP is a custom thematic management information system for urban and rural planning. In order to solve the problems in the management and manipulation of massive and multi-source heterogeneous spatial data, the concept of One Map was introduced in this paper firstly. And then, we de- 
signed and developed a URPSIP based on One Map and GIS. URPSIP could provide a integrate platform to deploy, browse, query, analyze and share planning information visually. Finally a case study in Wuhan proved that URPSIP is conducive to improve the management of urban and rural planning.

\section{REFERENCES}

[1] Yuan Q, Yi X, Wang X, et al. From Urban and Rural Integration to Real Urbanization: Rethinking the Development of The East Part of Nanhai. Urban Planning Forum, 2005(1):63-67.

[2] Zhang Y Z, Zhang Q W, Zhang Y. A Spatial Database Management System for Urban and Rural Planning. Applied Mechanics \& Materials, 2013, 411-414:357-361.

[3] Xin W. Urban Planning and Management Information Systems Analysis and Design Based on GIS, International Conference on Intelligent Information Technology Application. 2010:1440-1445.

[4] Kohsaka H. Applications of GIS to urban planning and management: Problems facing Japanese local governments. Geojournal, 2012, 52(3):271-280.

[5] Liu Q, Liu X. EXPLORATION AND PRACTICE OF "ONE MAP" PLANNING SYSTEM IN SHENZHEN[J]. City Planning Review, 2011.

[6] Ren Y, Liu Z, Hu L, et al. Design and implementation of spatial database system for seasonal influenza, 2011:1-6. 